\title{
Backward visual masking as a function of average uncertainty of the masking pattern
}

ROBERT E. FITZGERALD AND RICHARD KIRKHAM

UNIVERSITY OF WESTERN AUSTRALIA

The present experiment investigated informational content of the masking pattern in backward visual masking. Vertical or horizontal lines were flashed for from $4-17 \mathrm{msec}$. in a tachistoscope and were followed after a dark interval of 15 misec. by a random checkboard pattern presented for 150 msec. The probability of black squares in the checkboard was varied from 0.0 (all white) to 1.0 (all black). Accuracy of report of the orientation of the lines was a U-shaped func tion of the probability of black in the masking pattern. The similarity of this function to that of the average uncertainty of dichotomous distributions suggested a possible relationship between informational content and amount of masking.

When a briefly presented test stimulus is quickly followed by a second stimulus, backward visual masking may occur. To date, studies of backward visual masking in which pattern characteristics have been varied have been concerned with such aspects as parallelism of contours between the first and second stimuli (Werner, 1935, 1940; Pollack, 1965), relative degree of contrast (Pollack, 1965), general similarity between the two stimuli (Fehrer, 1965), and the amount of cross-hatching in the masking pattern (Schiller \& Wiener, 1963). Sperling (1964) suggests that backward visual masking by pattern results from interference with the information processing of the first pattern. If Sperling is correct, it might be predicted that maximum interference, and hence greatest masking, would occur when the masking pattern contained most information (i.e., average uncertainty). Garner (1962) states that the average uncertainty of dichotomous distributions is at a maximum when the probability of both categories (see Fig. 1) is 0.5 . In the present experiment, random black and white checkerboard patterns with the probability of black squares ranging from zero (i.e., all white) to 1.0 (i.e., all black) were used as masking patterns. If masking is related to average uncertainty of the masking pattern, then for a given matrix size it might be predicted that greatest masking would occur when the element probability of the masking pattern is $\mathbf{0 . 5}$, and least masking with the all black and all white masking patterns. (Amount of information would, of course, vary if the number of elements in the matrix was changed.)

\section{Method}

A Scientific Prototype Model GB three channel tachistoscope was used to present the patterns. The sequence of fields was Fixation, Field 1, Field 2, and return to Fixation. The white Fixation field contained four black marks which formed the corners of a 3 in. square set in the exact center of the field. The test patterns were presented for $4,7,10$ or 13 msec. (see below) in Field 1 , and the masking patterns for $150 \mathrm{msec}$. in Field 2. There was a dark interval of $15 \mathrm{msec}$. between the two fields. The brightness intensities of the fields were: Fixation, 2.3 ft.-L, Field 1, $2.8 \mathrm{ft} . \mathrm{L}$ and Field 2, $12.6 \mathrm{ft} . \mathrm{L}$. The distance from the eyes to Field 1 was 49 in. and to the other two, 48 in.

The test pattern was a 1 in. square containing five parallel lines $0.11 \mathrm{in}$. wide and $0.11 \mathrm{in}$. apart, drawn on a matt white card. The test pattern could be presented so that the lines were either vertical or horizontal.

The masking stimuli consisted of six 50 by 50 matrices each 2.5 in. square, in which the probabilities of blacked in elements were 0.0 (i.e., all white), $0.2,0.4,0.6,0.8$, and 1.0 (i.e., all black). The patterns were constructed from random matrices of 1 's and 0 's generated by a computer program. Two patterns represented each probability level, one being the inverted mirror image of the other. The test and masking stimuli nested centrally within the four fixation points.

The eight Ss were drawn from introductory Psychology courses at the University of Western Australia and were screened for the equivalent of a 13/16 Snellen score on the near acuity tests of the Bausch and Lomb Orthorater.

The following instructions were read to each S: "In this apparatus I am going to show you a series of patterns which will consist of sets of either horizontal or vertical lines. Your task will be to say whether they are horizontal or vertical. This discrimination will be made difficult by a second pattern coming on after the first. You may present the patterns to yourself by pressing this button once. If you look into this viewer, you will see a white field with four black marks forming the corners of a square. The horizontal or vertical lines will appear in the dead center of the square. (The two test patterns were shown to the Ss.) Now, I will give you some practice so you may see what the task is like. When I sound this buzzer you may press the button, and then say aloud whether the lines were vertical or horizontal. Remember to press the button only once. Do not worry if you think you are making mistakes. Do you have any questions?"

With the test stimulus field set at $10 \mathrm{msec}$., $\mathrm{S}$ received 12 practice trials using all possible combinations of test and masking stimuli once. After each presentation $\mathrm{S}$ was told whether his answer was correct. On the completion of the 12th practice trial, part 2 of the instructions were read: "That is the end of the practice series. During 
the actual experiment, I want you to write down either $\mathrm{V}$ or $\mathrm{H}$ in each square on this response sheet, depending on whether the lines were vertical or horizontal. Work across the page and if you are not sure, guess. There will be a short interval after each fifth pattern while I change the second pattern. Work quickly. The vertical and horizontal patterns will appear in a random order."

A further 20 trials using the $0.2,0.4,0.6$, and 0.8 masking patterns formed the basis for adjusting the time $\left(t_{1}\right)$ for which the testpatterm was presented. With $t_{1}$ set at $10 \mathrm{msec}$, the test patterns were presented randomly in horizontal and vertical positions in five consecutive trials for each masking pattern. If the total correct on these 20 trials was 13 or 14 then $t_{1}$ was retained at $10 \mathrm{msec}$. If the total correct exceeded 14 then $t_{1}$ was dropped to 7 msec., while if the total was less than 13 , $t_{1}$ was increased to $13 \mathrm{msec}$. Ss who fell into the last two categories were given another 20 trials, and the revised $t_{1}$ adjusted by $+3,0$, or -3 msec. according to identical criteria. On this basis, for the experimental trials, one $S$ operated at $t_{1}=4$ msec., three at $t_{1}=7$ msec., three at $t_{1}=10 \mathrm{msec}$, and one at $t_{1}=13 \mathrm{msec}$.

Each $S$ was given 220 experimental trials, in which the 0.0 and 1.0 masking patterns were presented 10 times each, and the other four masking patterns, 50 times each. The test stimulus was set at random in the vertical or horizontal position in each trial, but the masking stimulus was changed only after each fifth trial. For the first and last 30 presentations the order in which the masking stimuli were given was $0.4,0.2,0.8,0.6,1.0$ and 0.0 , while for the middle eight blocks of 20 presentations the order was $0.4,0.2,0.8$ and 0.6 . Overall, the test stimulus was presented an equal number of times in each orientation with each masking stimulus.

\section{Results}

The means and standard deviations of number of correct judgments for each of the six masking patterns are shown in Table 1. Figure 1 shows a plot of masking pattern probability against number of judgments incorrect. The curve was fitted using the Chebychev polynomial technique. Maximum masking occurred with the 0.4 and 0.6 probability patterns, and zero masking with the 0.0 and 1.0 probability patterns.

Table 1. Means and standard deviations of number of judgments correct for each of the six masking pattem probabilities.

\begin{tabular}{llc} 
P black & Mean & Standard Deviation \\
\hline 0.0 & 50.00 & 0.00 \\
.2 & 36.75 & 5.57 \\
.4 & 30.38 & 4.47 \\
.6 & 29.00 & 6.69 \\
.8 & 34.38 & 4.98 \\
1.0 & 50.00 & 0.00 \\
\hline
\end{tabular}

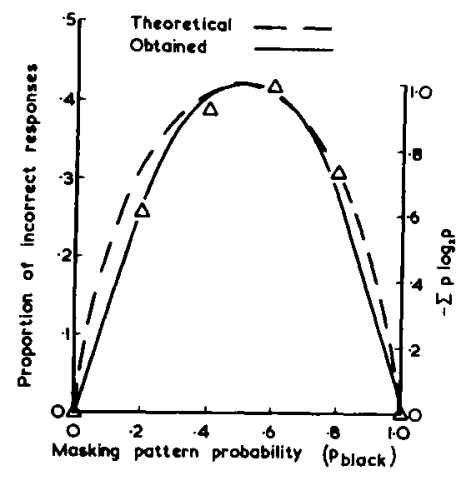

Fig. 1. Amount of backward masking with masking patterns containing different probabilities of black.

\section{Discussion}

Comparison of the plot of amount of masking against probability of black in the masking pattern with that of average uncertainty ( $-\Sigma$ plogp) against probability (Fig. 1) shows that the two functions are almost identical. The similarity of the two functions strongly suggests that backward visual masking is related to the amount of information (average uncertainty) in the masking pattern.

However, the present results could be specific to the stimulus patterns used, rather than to their informational content. The effects of only one matrix size (50 by 50 ) and only one size of matrix element (0.05 sq in.) were investigated. There are many other ways of varying information content (see Garner, 1962), for example, by varying the number of elements in the matrix. If masking is related to information then as the number of elements in the matrix is increased the amount of masking would also increase.

\section{References}

Fehrer, E. Contribution of perceptual segregation to the relationship between stimulus similarity and backward masking. Percept. mot. Skills, 1965, 21, 27-33.

Garner, W. R. Uncertainty and structure as psychological concepts. New York: Wiley, 1962.

Pollack, R. H. Effects of figure-ground contrast and contour orientation on figural masking. Psychon. Sci., 1965, 2, 369-370.

Schiller, P. H., \& Wiener, M. Monoptic and dichoptic masking. $J$. exp. Psychol., 1963, 66, 386-393.

Sperling, G. What visual masking can tell us about temporal factors in perception. Acta Psychol., 1964, 23, 199-200.

Wemer, H. Studies on contour: I. Qualitative analysis, Amer. J. Psychol., 1935, 47, 40-64.

Wemer, H. Studies on contour strobostereoscopic phenomena. Amer. J. Psychol., 1940, 53, 418-422. 